\title{
Dual immune checkpoint blockade for non-small cell lung cancer patients with PD-L1 high expression: calling an end?
}

\author{
Ling Peng ${ }^{1}$, Justin Stebbing ${ }^{2}$, Fei Liang ${ }^{3}$, Yang Xia ${ }^{4}$ \\ ${ }^{1}$ Department of Respiratory Disease, Zhejiang Provincial People's Hospital, Hangzhou, China; ${ }^{2}$ Division of Cancer, Department of Surgery and \\ Cancer, Imperial College London, London, UK; ${ }^{3}$ Department of Biostatistics, Zhongshan Hospital, Fudan University, Shanghai, China; ${ }^{4}$ Key \\ Laboratory of Respiratory Disease of Zhejiang Province, Department of Respiratory and Critical Care Medicine, The Second Affiliated Hospital, \\ School of Medicine, Zhejiang University, Hangzhou, China \\ Correspondence to: Yang Xia. Department of Respiratory and Critical Care Medicine, Second Affiliated Hospital, Zhejiang University School of \\ Medicine, Hangzhou 310009, China. Email: yxia@zju.edu.cn.
}

Submitted Jul 26, 2021. Accepted for publication Aug 30, 2021.

doi: $10.21037 /$ tlcr-21-650

View this article at: https://dx.doi.org/10.21037/tlcr-21-650

We read with interest the KEYNOTE-598 trial (1), which did not meet its primary endpoints and was terminated early. Additional ipilimumab failed to amplify the efficacy of pembrolizumab in the 'tumor proportion score (TPS) high' population which could be attributed to several reasons.

First, the clinical role of ipilimumab in addition to PD-1 antibody in the first line treatment for non-small cell lung cancer (NSCLC) is marginal, as previously demonstrated by CheckMate 227 part 1 (2). However, discernment of whether combining an immune checkpoint inhibitor (ICI) with chemotherapy is more efficient than ICI monotherapy in a PD-L1 high population is of great importance. Evidence from a meta-analysis showed that although objective response rate (ORR) as well as progression-free survival (PFS) were improved in the combination group, the overall survival (OS) benefit presented was identical (3). While an anti-PD-1 antibody plus chemotherapy performs better than an anti-PD-L1 antibody plus chemotherapy for first line NSCLC regardless of PD-L1 expression (4), the difference of anti-PD-1 versus anti-PD-L1 monotherapy in a PD-L1 high expression population is not significant, with results from TC3/IC3 of IMpower-110 for OS hazard ratio (HR) 0.59 [95\% confidence interval (CI): 0.40 to 0.89] (5) and EMPOWER-Lung 1 showing an OS HR of 0.57 (95\% CI: 0.42 to 0.77 ) (6). Although cross-trial comparisons are fraught with difficulties, we do have similar numerical median survival data of ICI monotherapy for this population. Hence, with similar PFS and OS results of the KEYNOTE-024 and KEYNOTE-598 trials, pembrolizumab monotherapy would remain the standard of care for the first line setting of PD-L1 high expressors at the current stage.

Second, the toxicity profile of ipilimumab impacts the adverse event profile of dual ICI blockade. Adding ipilimumab was associated with a higher rate of grade 3-5 adverse events for NSCLC patients in KEYNOTE-598. With prior experience using ipilimumab in NSCLC, KEYNOTE-598 contributed evidence to the role of antiCTLA-4 into the first line treatment of NSCLC. According to data from the use of durvalumab plus tremelimumab in the MYSTIC trial (7), the toxicity of CTLA-4 inhibitors is not restricted to ipilimumab. In balancing the survival benefit and toxicity risk of PD-L1 high expressors, dual ICI blockade of PD-L1/CTLA-4 might not be an optimal choice. Toxicity profile of ipilimumab could be reduced with lower dose or longer interval of treatment, indicating the dedicate balance of efficacy versus toxicity of anti-CTLA-4 antibody.

Third, the underlying biologic mechanisms might explain the failure of KEYNOTE-598. The PD-1/PDL1 pathway is a major immune-related mechanism in these cancers, but the tumor immune microenvironment

^ ORCID: 0000-0002-1359-4982. 
of patients with NSCLC with high PD-L1 expression may be 'inflammatory'. Thus, up-stream blockage of CTLA4 may not bring additional benefits for this population. Retrospective studies of NSCLC with very-high PDL1 expression levels (defined as $90 \%$ and above) have suggested PD-1 inhibitor monotherapy treatment rather than in combination with chemotherapy (8). Therefore, stratification measures might ensure randomized groups are evenly balanced by various PD-L1 levels in KEYNOTE-598. In KEYNOTE-598, PD-L1 expression $>90 \%$ vs. $50-90 \%$ is not stratified, therefore there was no information regarding survival of these groups. The expression levels of PD-L1 remain the most common predictive biomarkers of the clinical benefits from PD-L1 inhibitors in the real world; although, whether high PD-L1 expression would predict clinical efficacy of PD-L1/CTLA4 dual blockade is not known.

Finally, which is the optimal treatment strategy for NSCLC patients of high PD-L1 expression? Monotherapy of PD-L1 inhibitors works well for PD-L1 high expressors, however, it would be ideal to further prolong the median OS of 26.3 months that was achieved with pembrolizumab monotherapy, as reported in KEYNOTE-024 (9). The KEYNOTE-598 trial did not answer the question of whether adding chemotherapy to immunotherapy for PDL1 high expressors would lead to further survival benefit. The ongoing trials of INSIGNA (NCT03793179) and PERSEE (NCT04547504) will provide further clarity.

The question remains whether there is any role for other dual checkpoint blockade other than CTLA-4 in NSCLC patients with high PD-L1 expression. The phase 2 trial CITYSCAPE analyzed an anti-TIGIT ( $T$ cell immunoreceptor with immunoglobulin and ITIM domain) antibody plus atezolizumab as a dual checkpoint blockade combination (10). Therein, PD-L1 high expressors appeared to benefit from adding anti-TIGIT antibody with an OS HR of 0.30 (95\% CI: 0.15 to 0.61 ) in this subgroup, while patients with PD-L1 expression of $1-49 \%$ did not benefit from this combination, indicating a potential role of TIGIT for PD-L1 high expression NSCLC patients. The phase III SKYSCRAPER-01 trial (NCT04294810) will help to elucidate these results.

In conclusion, although not practice changing, the failure of KEYNOTE-598 provides insights for the current landscape of NSCLC treatment. Caution should be exercised when creating future designs for dual immunotherapy oncology clinical trials without promising results from exploratory phase II trials.

\section{Acknowledgments}

Funding: None.

\section{Footnote}

Provenance and Peer Review: This article was a standard submission to the journal. The article has undergone external peer review.

Conflicts of Interest: All authors have completed the ICMJE uniform disclosure form (available at https://dx.doi. org/10.21037/tlcr-21-650). Professor JS, the Editor-inChief of Oncogene has sat on SABs for Vaccitech, Heat Biologics, Eli Lilly, Alveo Technologies, Pear Bio, Agenus, Equilibre Biopharmaceuticals, Graviton Bioscience Corporation, Celltrion, Volvox, Certis Oncology Solutions, Greenmantle, Zedsen, Bryologyx and Benevolent AI. He has consulted with Lansdowne partners and Vitruvian. He sits on the Board of Directors for Xerion and BB Biotech Healthcare Trust PLC. The other authors have no conflicts of interest to declare.

Etbical Statement: The authors are accountable for all aspects of the work in ensuring that questions related to the accuracy or integrity of any part of the work are appropriately investigated and resolved.

Open Access Statement: This is an Open Access article distributed in accordance with the Creative Commons Attribution-NonCommercial-NoDerivs 4.0 International License (CC BY-NC-ND 4.0), which permits the noncommercial replication and distribution of the article with the strict proviso that no changes or edits are made and the original work is properly cited (including links to both the formal publication through the relevant DOI and the license). See: https://creativecommons.org/licenses/by-nc-nd/4.0/.

\section{References}

1. Boyer M, Şendur MAN, Rodríguez-Abreu D, et al. Pembrolizumab plus ipilimumab or placebo for metastatic non-small-cell lung cancer with PD-L1 tumor proportion score $\geq 50 \%$ : randomized, double-blind phase III KEYNOTE-598 study. J Clin Oncol 2021;39:2327-38.

2. Ramalingam SS, Ciuleanu TE, Pluzanski A, et al. Nivolumab+ ipilimumab versus platinum-doublet chemotherapy as first-line treatment for advanced nonsmall cell lung cancer: three-year update from CheckMate 
227 Part 1. J Clin Oncol 2020;38:abstr 9500.

3. Zhou Y, Lin Z, Zhang X, et al. First-line treatment for patients with advanced non-small cell lung carcinoma and high PD-L1 expression: pembrolizumab or pembrolizumab plus chemotherapy. J Immunother Cancer 2019;7:120.

4. Dafni U, Tsourti Z, Vervita K, et al. Immune checkpoint inhibitors, alone or in combination with chemotherapy, as first-line treatment for advanced non-small cell lung cancer. A systematic review and network meta-analysis. Lung Cancer 2019;134:127-40.

5. Jassem J, Herbst RS, de Marinis F, et al. IMpower110: Clinical safety in a phase III study of atezolizumab (atezo) monotherapy (mono) vs platinum-based chemotherapy (chemo) in first-line non-small cell lung cancer (NSCLC). J Clin Oncol 2020;38:e21623.

6. Sezer A, Kilickap S, Gümüş M, et al. Cemiplimab monotherapy for first-line treatment of advanced nonsmall-cell lung cancer with PD-L1 of at least 50\%: a multicentre, open-label, global, phase 3, randomised, controlled trial. Lancet 2021;397:592-604.

7. Rizvi NA, Cho BC, Reinmuth N, et al. Durvalumab with or without tremelimumab vs standard chemotherapy in first-line treatment of metastatic non-small cell lung cancer: the MYSTIC phase 3 randomized clinical trial. JAMA Oncol 2020;6:661-74.

8. Aguilar EJ, Ricciuti B, Gainor JF, et al. Outcomes to first-line pembrolizumab in patients with non-small-cell lung cancer and very high PD-L1 expression. Ann Oncol 2019;30:1653-9.

9. Brahmer JR, Rodriguez-Abreu D, Robinson AG, et al. LBA51 KEYNOTE-024 5-year OS update: first-line (1L) pembrolizumab (pembro) vs platinum-based chemotherapy (chemo) in patients (pts) with metastatic NSCLC and PDL1 tumour proportion score (TPS) $\geq 50 \%$. Ann Oncol 2020;31:S1181-2.

10. Rodriguez-Abreu D, Johnson ML, Hussein MA, et al. Primary analysis of a randomized, double-blind, phase II study of the anti-TIGIT antibody tiragolumab (tira) plus atezolizumab (atezo) versus placebo plus atezo as first-line (1L) treatment in patients with PD-L1-selected NSCLC (CITYSCAPE). J Clin Oncol 2020;38:abstr 9503.

(English Language Editor: J. Jones)
Cite this article as: Peng L, Stebbing J, Liang F, Xia Y. Dual immune checkpoint blockade for non-small cell lung cancer patients with PD-L1 high expression: calling an end? Transl Lung Cancer Res 2021;10(9):3858-3860. doi: 10.21037/tlcr21-650 